S3. Ministry of Agriculture - National Agricultural Laboratories, Nairobi, Kenya.

Mode, C.J., and H.F. Robinson. 1959. Pleiotropism and the genetic variance and covariance. Biometrics 15:518-537.

Niehaus, M.H., and R.C. Pickett. 1966. Heterosis and combining ability in a diallel cross in Sorghum vulgare Pers. Crop Sci. 6:33-36.

Patanothai, A., and R.E. Atkins. 1974. Yield stability of single crosses and three-way hybrids of grain sorghum. Crop Sci. 14:287-290.

Reich, V.H., and R.E. Atkins. 1970. Yield stability of four population types of grain sorghum, Sorghum bicolor (L.) Moench, in different environments. Crop Sci. 10:511-517.

Ross, W.M. 1965. Yield of grain sorghum (Sorghum vulgare Pers.) hybrids alone and in blends. Crop Sci. 5:593-594.

Schnell, F.W., and H.C. Becker. 1986. Yield and yield stability in a balanced system of widely differing population structures in Zea mays L. Plant Breeding 97:30-38.

Snedecor, G.W., and W.G. Cochran. 1980. Statistical methods. 7th ed.. The Iowa State University Press, Ames.

Stelling, D., E. Ebmeyer, and W. Link. 1994. Yield stability in faba bean, Vicia faba L. 2. Effects of heterozygosity and heterogeneity. Plant Breeding 112:30-39.

Tukey, J.W. 1949. One degree of freedom for non-additivity. Biometrics 5:232-242.
Ud-Din, N., B.F. Larver, and A.C. Clutter. 1992. Genetic analysis and selection for wheat yield in drought stressed and irrigated environments. Euphytica 62:89-96.

Utz, H.F. 1993. PLABSTAT: A computer program for the statistical analysis of plant breeding experiments. Institute of Plant Breeding, Seed Science, and Population Genetics, University of Hohenheim, Stuttgart, Germany.

Virk, D.S., and B.K. Mangat. 1991. Detection of cross over genotype $\times$ environment interactions in pearl millet. Euphytica 52:193-199.

Wahle, G., and H.H. Geiger. 1978. Vergleich der phänotypischen Stabilität von Inzuchtlinien, Einfachhybriden und Populationen bei Winterroggen. Z. Pflanzenzüchtg. 80:211-222.

Walulu, R.S., D.T. Rosenow, D.B. Wester, and H.T. Nguyen. 1994. Inheritance of the stay green trait in sorghum. Crop Sci. 34:970-972.

Ward, J.H. 1963. Hierarchical grouping to optimize an objective function. J. Am. Stat. Assoc. 58:236-244.

Watson, S.H., I.H. DeLacy, D.W. Podlich, and K.E. Basford. 1996. GEBEI. An analysis package using agglomerative hierarchical classificatory and SVD ordination procedures for genotype $\times$ environment data. Research Report \#57, Department of Mathematics, The University of Queensland, Brisbane, Australia.

\title{
Economic Efficiency of One Cycle of Marker-Assisted Selection
}

\author{
L. Moreau,* S. Lemarié, A. Charcosset, and A. Gallais
}

\begin{abstract}
The efficiency of molecular markers to improve genetic prediction has been proved by many studies. Nevertheless, the additional cost due to marker genotyping is seldom considered in the comparison between marker-assisted selection (MAS) and phenotypic selection. In the context of plant breeding, the relative cost efficiency of MAS in the first cycle of selection is evaluated through an analytical approach taking into account the effect of the experimental design (population size, number of trials, and replications per trial) on quantitative trait loci (QTL) detection. The same global cost is assumed for both methods. In a first step, the optimal allocation of the experimental resources is studied for each method before comparing them at their optimum. For traits sensitive to genotype $\times$ environment interactions, unreplicated trials are optimal for both methods but the optimal number of trials is different. It increases with the investment for phenotypic selection while it becomes nearly equal to one for MAS. The loss of efficiency due to non-optimal designs is evaluated. The expected economic return of MAS compared with phenotypic selection decreases with the cost of genotyping. When this cost is high, MAS interest is limited to traits with a low heritability, provided that the investment is high enough to evaluate a large population size, which is the necessary condition to explain with markers a large part of genetic variation. The maximal genotyping cost that is acceptable for MAS to be efficient is given for different values of investment and trait heritability.
\end{abstract}

$\mathrm{F}_{\mathrm{i}}$ OR THE LAST DECADE, rapid progress has been made in molecular marker techniques. For many species, the number of polymorphic markers is now sufficient

L. Moreau, A. Charcosset, A. Gallais, I.N.R.A.-U.P.S.-I.N.A.-P.G., Station de Génétique Végétale, Ferme du Moulon, F-91190 Gif-surYvette, France; S. Lemarié, I.N.R.A., S.E.R.D. Département d'Economie et de Sociologie Rurales, Université Pierre Mendès France, BP 47, F-38040 Grenoble cedex 9, France. Recieved 19 Jan. 1999. *Corresponding author (moreau@moulon.inra.fr).

Published in Crop Sci. 40:329-337 (2000). to build dense maps and to localize individual QTL. Once marker-QTL associations have been detected, they can be used to improve the prediction of genetic values. This use of markers in selection schemes has received extensive interest in the recent past. Lande and Thompson (1990) proposed to identify marker-QTL associations by multiple regression of phenotype on marker type and to select individuals on an index combining phenotype and molecular value predicted with markers. The efficiency of this method of markerassisted selection (MAS) relative to purely phenotypic selection has been widely studied, mainly in the case of populations derived from the cross of two inbred lines, through analytical approaches (Lande and Thompson, 1990; Luo et al., 1997; Moreau et al., 1998) and simulations (Zhang and Smith, 1992, 1993; Gimelfarb and Lande, 1994, 1995; Whittaker et al., 1995, 1997; Hospital et al., 1997). All these theoretical studies concluded that in many situations, MAS could be more efficient than phenotypic selection.

Despite these promising results, to our knowledge, few MAS experiments have been conducted with the approach proposed by Lande and Thompson (1990). This is certainly partly due to the cost of MAS experiments, which require both phenotypic and molecular evaluation. In all the theoretical studies mentioned above, the problem of the additional cost due to marker evaluation is not taken into account. Both methods of selection are assumed to be conducted in the same agricultural conditions, that is to say, assuming equal population sizes and identical experimental designs. In this situation, the additional cost associated with the marker evaluation is ignored, which leads to overestimating the

Abbreviations: DH, doubled haploids; MAS, marker-assisted selection; QTL, quantitative trait locus. 
interest of MAS compared with the phenotypic selection. Recently, Xie and Xu (1998) evaluated analytically the relative efficiency of different strategies of MAS, and incorporated the costs associated with measuring phenotype and scoring marker loci into the objective function, to maximize the gain per cost unit. Nevertheless, when estimating the genetic gain associated with MAS, the percentage of variance associated with the detected QTLs was assumed to be a known and fixed parameter. This would only be the case if the QTL detection and the estimation of the QTL effects were done in an infinite population. The same assumption was made by Knapp (1998) who compared the number of progenies that must be evaluated in phenotypic selection or MAS to retain a given proportion of superior genotypes. It is now well established that using finite populations generates important uncertainties with respect to QTL detection and effect estimation (Beavis, 1994). Thus, the size of the population under selection and also the experimental design, which affects the heritability of the trait, must be taken into account since they are deciding parameters of QTL detection and consequently MAS efficiency (Lande and Thompson, 1990; Zhang and Smith, 1992, 1993; Gimelfarb and Lande, 1994; Whittaker et al., 1995, 1997; Hospital et al., 1997; Moreau et al., 1998).

To be fair, MAS and phenotypic selection must be compared at their optimum for a given cost attributed to the selection process and for a given number of selected entries at the end of the cycle. For the same cost, phenotypic selection, which does not require marker evaluation, allows breeders to increase the population size and thus the intensity of selection compared with the one used for MAS. Alternatively, breeders can improve the phenotypic evaluation and thus the heritability of the trait by doing replications, as is usually done in plant breeding. This possibility of doing replications requires (i) either populations composed of fixed genotypes like inbred lines, or (ii) progeny tests, often used in QTL detection experiments (for instance, F2 population evaluated through the mean performance of derived F3 families). Instead of doing replications, other methods of prediction based on performances of relatives (classical full-sib or half-sib schemes) or on associated traits may be more adapted for improving the genetic gains for MAS and phenotypic selection (see Lande and Thompson, 1990) but will not be considered here. When it is possible to replicate individuals, several allocations of the experimental resources are possible (population size, number of locations, replications per location) with a fixed experimental cost. Before comparing the efficiency of MAS and phenotypic selection, the allocation of experimental resources, which leads to the highest genetic gain, must be determined for each method.

In the plant breeding context, the optimization of the number of replications and the population size in experiments of phenotypic selection has already been studied in several papers (Bos, 1983; Wricke and Weber, 1986; Gauch and Zobel, 1996). This has not yet been done in the MAS context. The determination of the optimal design requires one to be able to predict the genetic gain for a large range of situations in order to determine the one which gives the highest genetic progress. As simulations are too time consuming, the ideal is to use an analytical approach. In this paper, we use the approach developed by Moreau et al. (1998) which, unlike the formulation of Lande and Thompson (1990), fully takes into account the effect of population size. Moreover it was shown to give results consistent with those obtained by simulation with a more complex genetic model (Hospital et al., 1997).

Extending the study of Moreau et al. (1998), the aim of this paper is to investigate (i) the optimal allocation of experimental resources for MAS and phenotypic selection and, then (ii) to evaluate the domains where MAS is cost efficient. This work is restricted to the plant breeding context: we assume that the population is derived from a cross between two inbred lines and that the heritability of the trait can be improved by replicating each entry several times, in different field trials or within the same trial.

\section{METHODS}

\section{Phenotypic and Marker-Assisted Selection}

We consider that the aim of the selection is to improve the mean value of the population over several environments. For a pure phenotypic selection, the genetic value of an individual, $i$, of a population sample can be simply estimated from its average performance $\left(P_{i}\right)$ within the whole experimental design. If each individual $i$ is evaluated in $L$ different trials, corresponding to different locations, and if its evaluation is replicated $K$ times within each trial (divided, for instance, in $K$ complete blocks), then:

$$
\hat{G}_{i}^{\mathrm{P}}=P_{i}=\frac{1}{K L} \sum_{i=1}^{L} \sum_{k=1}^{K} p_{i k}
$$

where $p_{\text {ilk }}$ is the $k$ th performance of $i$ evaluated within the trial $l$. The basic experimental unit used to evaluate $p_{\text {ilk }}$ will be further referred to as a plot. Each plot is sown with only one genotype $i$ but may contain one or several plants. For instance, in most species the yield performance of a genotype is evaluated through the bulked harvest of several plants.

Following Lande and Thompson (1990), in MAS, individuals are selected on an index including both phenotype and marker information:

$$
\hat{G}_{i}^{\mathrm{M}}=\hat{b}_{\mathrm{p}} P_{i}+\hat{b}_{\mathrm{m}} M_{i}
$$

where $P_{i}$ is the average performance of $i$ (see above), $M_{i}$ is the sum of the effects of the markers associated to the trait and is called Molecular score, $\hat{b}_{\mathrm{p}}$ and $\hat{b}_{\mathrm{m}}$ are the weight coefficients of the index. $M_{i}=\sum_{q} \hat{a}_{q} \theta_{i q}$, where $q$ indicates markers significantly associated with the trait of interest, $\hat{a}_{q}$ is the estimated additive effect associated with one of the parental alleles at marker $q$, and $\theta_{i q}$ is a dummy variable that takes the value $-1,0$, or 1 according to the number of copies of the given parental allele at marker $q$ is 0,1 , or 2 , respectively. With the aim of predicting the mean value of the progeny of a given individual in the next generation, only additive effects need to be evaluated. The weight coefficients that maximize the genetic gain are defined by:

$$
\hat{b}_{p}=\frac{h_{\mathrm{b}}^{2}-\hat{R}_{\mathrm{m}}^{2}}{1-\hat{R}_{\mathrm{m}}^{2}} \quad \text { and } \quad \hat{b}_{\mathrm{m}} \frac{1-h_{\mathrm{b}}^{2}}{1-\hat{R}_{\mathrm{m}}^{2}}
$$


where $\hat{R}_{\mathrm{m}}^{2}$ is the estimated percentage of phenotypic variance associated with markers and $h_{\mathrm{b}}^{2}$ is the broad sense heritability associated with the average performance $P_{i}$ in the experimental design.

$$
h_{\mathrm{b}}^{2}=\frac{\sigma_{\mathrm{G}}^{2}}{\sigma_{\mathrm{G}}^{2}+\frac{\sigma_{\mathrm{GE}}^{2}}{L}+\frac{\sigma_{\mathrm{e}}^{2}}{L K}}
$$

$\sigma_{\mathrm{G}}^{2}$ is the additive genetic variance common to all the environments, $\sigma_{\mathrm{GE}}^{2}$ is the variance of the genotype $\times$ environment interaction and $\sigma_{\mathrm{e}}^{2}$ is the residual variance due to experimental error. We can define another heritability that will be further mentioned as individual heritability and noted $h_{i}^{2}$ :

$$
h_{i}^{2}=\frac{\sigma_{\mathrm{G}}^{2}}{\sigma_{\mathrm{G}}^{2}+\sigma_{\mathrm{GE}}^{2}+\sigma_{\mathrm{e}}^{2}}
$$

which represents the proportion of the phenotypic variation, observed at the level of an individual plot, due to genetic effects common to all the environments. This parameter does not depend on the experimental design and is specific to the trait.

\section{Definition of Economic Parameters}

Assuming that in phenotypic selection, the experimental costs are directly proportional to the number of plots, the total experimental resources $I^{\mathrm{P}}$ required to achieve one cycle of selection are: $I^{\mathrm{P}}=N^{\mathrm{P}} L^{\mathrm{P}} K^{\mathrm{P}} C$, where $N^{\mathrm{P}}$ is the total number of the genotypes of the population sample evaluated, $C$ is the individual cost needed to evaluate the phenotypic value of one genotype in one plot (i.e., $p_{\text {ilk }}$ ), $L^{\mathrm{P}}$ and $K^{\mathrm{P}}$ are the number of trials and the number of replications per trial, defined above. The superscript $\mathrm{P}$ refers to the phenotypic selection.

For MAS, the investment $\left(I^{\mathrm{M}}\right)$ needed to achieve one cycle is $I^{\mathrm{M}}=N^{\mathrm{M}}\left(L^{\mathrm{M}} K^{\mathrm{M}} C+C_{\text {mark }}\right)$, where $N^{\mathrm{M}}, L^{\mathrm{M}}$ and $K^{\mathrm{M}}$ are the $N, L$, and $K$ parameters for MAS (indicated with the superscript M). $C_{\text {mark }}$ is the cost associated with the genotyping of one individual. This quantity can be expressed as an equivalent number of plots that could be done with the same experimental resources: $C_{\text {mark }}=C R_{\mathrm{C}}$, where $R_{\mathrm{C}}$ is the relative cost of genotyping one individual compared with the cost of evaluating one individual in one plot. Obviously, the cost of genotyping depends on the number of markers and on the individual cost of one marker-data point. In the first cycle of selection, marker-QTL associations have to be determined, which requires enough markers to have good coverage of the genome. Because this number depends on the genome length, for sake of simplicity, we did not evaluate the effect of this number and only considered in our formulations the global genotyping cost. With these notations, all the costs can be expressed in field plot unit. $I_{0}^{\mathrm{P}}=I^{\mathrm{P}} / C=N^{\mathrm{P}} L^{\mathrm{P}} K^{\mathrm{P}}$ and $I_{0}^{\mathrm{M}}=I^{\mathrm{M}} / C=N^{\mathrm{M}}\left(L^{\mathrm{M}}\right.$ $\left.K^{\mathrm{M}}+R_{\mathrm{C}}\right)$. The comparison of the two methods for given fixed experimental resources $\left(I_{0}\right)$ leads to the relationship:

$$
I_{0}=N^{\mathrm{P}} L^{\mathrm{P}} K^{\mathrm{P}}=N^{\mathrm{M}}\left(L^{\mathrm{M}} K^{\mathrm{M}}+R_{\mathrm{C}}\right)
$$

As $R_{\mathrm{C}} \neq 0$, the above relation implies that the two methods are conducted with different sets of $L, K$, and $N$ values. For a given $I_{0}$ and $R_{\mathrm{C}}$, the problem is to determine the values of $N, L$, and $K$ that give the highest genetic gain for each method.

\section{Evaluation of the Genetic Gains}

Assuming that the selected trait is normally distributed in the population, the expected genetic gain after one cycle of phenotypic selection is equal to (Falconer, 1981) $\Delta G_{\mathrm{P}}=i h_{\mathrm{b}} \sigma_{\mathrm{G}}$, where $i$ is the intensity of selection in standard unit, $h_{\mathrm{b}}$ is the square root of the broad sense heritability defined above, and $\sigma_{\mathrm{G}}$ is the genetic standard deviation. In an infinite population, $i$ is equal to $z / p$, where $z$ is the height of the ordinate at the point of truncation of a normal distribution and $p$ is the proportion of selected individuals. The broad sense heritability $h_{b}^{2}$ depends on the experimental design $\left(L^{\mathrm{P}}\right.$ and $\left.K^{\mathrm{P}}\right)$ and on the proportions $\sigma_{\mathrm{G}}^{2} / \sigma_{\mathrm{e}}^{2}$, and $\sigma_{\mathrm{GE}}^{2} / \sigma_{\mathrm{e}}^{2}$, which are specific to the trait.

For MAS, the analytical expression of the expected genetic gain is more complex and implies more parameters. Among them, the population sample size has a major effect. It strongly affects the power of QTL detection and the precision of the estimation. In finite population samples, when QTL detection and genetic prediction are made on the same sample, the selection of the markers included in the index (Eq. [2]) leads to an overestimation of the effects associated with markers (Lande and Thompson, 1990; Beavis, 1994). This bias of selection is difficult to correct (Whittaker et al., 1997; Melchinger et al., 1998) and was taken into account to evaluate MAS efficiency (Moreau et al., 1998). Other parameters influence the MAS genetic gain: the genetic determinism of the trait (the number of QTLs involved, their localization, the distribution of their effects), the molecular map (the number of markers and their position relative to QTLs), the method of QTL detection used, and the rule for the selection of markers in the index.

To take these parameters into account, we used the approach of Moreau et al. (1998) (see this paper for a full description and discussion of the model). We consider that the genetic variability of the trait is explained by $x_{1}$ independent QTLs of equal effect, with no epistasis. Two genetic models are considered: $x_{1}=5$ or 10 QTLs. Each QTL is linked with a single marker. Other markers $\left(30-x_{1}\right)$, unlinked with QTL, are added to the model to simulate possible false detections. The total number of 30 markers was chosen to roughly correspond to 10 chromosomes and three nearly independent markers located on each. Marker-QTL associations are detected by a simple regression, taking a type I risk level equal to $5 \%$, chosen following the results of Moreau et al. (1998). The rate of recombination (noted $r$ ) between each QTL and its linked marker is assumed to be the same for each QTL. This parameter determines $m^{2}$, the maximum percentage of genetic variance that can be explained by markers. In a population composed of doubled haploids $(\mathrm{DH}), m^{2}$ is equal to $(1-2 r)^{2}$. In this study, we do not address the problem of the optimization of the map density. Thus, we consider a fixed marker-QTL distance, corresponding to an $m^{2}$ value equal to 0.8 . In a DH population, this value is obtained with an average density of one marker every 20 centimorgans, which was shown to be nearly optimal (Gimelfarb and Lande, 1994; Darvarsi et al., 1994). Moreau et al. (1998) highlighted that a small number of QTLs with equal effects provides a good approximation of the results obtained with more QTLs and a geometric distribution of their effects, corresponding to the same equivalent number of QTL (as defined by Lande, 1981, and further noted $\left.x_{\mathrm{e}}\right)$. If QTLs have variable contributions to the genetic variance, the equivalent number of QTLs is expected to be rather small (between 3 and 20) (Chevalet, 1994). In many studies addressing the potential benefit of MAS by means of simulations, an equivalent number of 10 QTLs is assumed (Gimelfarb and Lande, 1994; Hospital et al., 1997). This model was shown to give consistent results with such simulations conducted with a more realistic genetic model and marker allocation, and a multiple regression for the QTL detection. Thus, the model considered here is certainly too simple, but rapidly yields results that can reasonably be extrapolated to more realistic situations, at least in the first cycle. 
In all this work, for both methods of selection, we consider that a fixed number of individuals $\left(n_{\mathrm{s}}\right)$ is selected (i.e., the same effective genetic size). Following Eq. [6], the proportion of selected individuals is $p^{\mathrm{P}}=n_{\mathrm{s}} / N^{\mathrm{P}}=K^{\mathrm{P}} L^{\mathrm{P}}\left(n_{\mathrm{s}} / I_{0}\right)$ for phenotypic selection, and $p^{\mathrm{M}}=\left(K^{\mathrm{M}} L^{\mathrm{M}}+R_{\mathrm{C}}\right)\left(n_{\mathrm{s}} / I_{0}\right)$ for MAS. The results that will be presented are obtained with a fixed number of selected individuals $n_{\mathrm{s}}$ equal to 10 . This number represents a compromise between short term selection efficiency and the risk of genetic drift in the long term. As the population sample size is limited when the investment is low, the intensity of selection $i$ corresponding to the $p$, found in the table given by Falconer (1981) for an infinite population, was corrected by the approximate formulation given by Burrows (1972) to take the population sample size into account: $i_{N}=i-(N-$ $\left.n_{\mathrm{s}}\right) /\left[2 n_{\mathrm{s}}(N+1) i\right]$, where $i$ and $i_{N}$ are the intensities of selection for an infinite population and for population sample size of $N$, respectively.

\section{Optimization of the Experimental Parameters-Relative Efficiency of MAS}

For a given trait (characterized by $h_{i}^{2}$ and $\sigma_{\mathrm{GE}}^{2} / \sigma_{\mathrm{e}}^{2}$ ) and given values of $I_{0}$ and $R_{\mathrm{C}}$, we first determine the combination of the three parameters $N, K$ (between 1 and 25), and $L$ (between 1 and 25), which maximizes the genetic gain. Because a strict analytical solution of this problem requires complex mathematical derivations, the determination of the optimal allocation of the experimental resources is made numerically by comparing the genetic gain obtained with different experimental designs. The values $(L, K)$ leading to the highest genetic gain are considered as optimal for a given method. The genetic gains $\left(\Delta G_{\mathrm{M}}\right.$ and $\left.\Delta G_{\mathrm{P}}\right)$ obtained for each method with an optimal allocation of the same investment are later compared. The relative cost efficiency of MAS (further noted $R E$ ) is defined by the ratio:

$$
R E=\frac{\Delta G_{\mathrm{M}}}{\Delta G_{\mathrm{P}}}
$$

The program was implemented in FORTRAN. For MAS, the genetic gain evaluation was performed by the statistical facilities of SAS (1988). Because these two programs were not easy to connect, MAS genetic gains were first evaluated for a large range of values of $N$ and $h_{b}^{2}$ and stocked in a data file. The genetic gain for a particular set of values $\left(N, h_{b}^{2}\right)$ was estimated by linear approximations between the genetic gains found in the data file that were evaluated with the nearest flanking values of these parameters.

\section{RESULTS \\ Optimal Allocation of the Experimental Resources}

For MAS and phenotypic selection, when non-zero genotype $\times$ environment interactions are considered in the model, the highest genetic gain is always obtained when the genotypes are evaluated only once $(K=1)$ in each field trial (results not shown). This conclusion already drawn by Wricke and Weber (1986) for a phenotypic selection appears to be also valid for MAS. When genotype $\times$ environment interactions are considered as null, there is no need to evaluate the population in several locations. For practical reasons, the best strategy is to put all the genotypes in the same trial $(L=1)$ and, if needed, to replicate them within the field trial.
As a consequence, for both methods of selection, all results presented can be interpreted either as (i) the optimal number of trials $(L)$ with one replication per trial if the trait is affected by genotype $\times$ environment interactions or (ii) the optimal number of replications $(K)$ within one trial if the trait is not affected by such interactions. For sake of simplicity, we will henceforth always use the term replication without mentioning whether it corresponds to replications in different trials or within the same one. The other consequence is that the results do not depend on the ratio $\sigma_{\mathrm{GE}}^{2} / \sigma_{\mathrm{e}}^{2}$, the only effective parameter being $h_{i}^{2}$.

The comparison of the results obtained for each method shows that the optimal number of replications is quite different for MAS and phenotypic selection (Fig. 1). For phenotypic selection (see graph a), when the individual heritability is high (above 0.7 ), doing replications does not sufficiently increase the broad sense heritability to compensate for the decrease in selection intensity associated with a smaller population sample size. For lower heritabilities, replications can increase genetic gain provided that the total investment is high enough. Thus, the optimal number of replications increases with the investment and decreases with the individual heritability. So, for very low individual heritabilities $\left(h_{i}^{2}<0.10\right)$, it is optimal to do a high number of replications (above 6) even with a medium investment (equivalent to 600 plots).

For MAS, the results were derived with a genetic model including $x_{\mathrm{e}}=5$ QTLs, but different values of the relative cost of genotyping $\left(R_{\mathrm{C}}\right.$ equal to 1,4 , or 8 ; Fig. 1b, c, d). One can first note that the optimal number of replications always increases as $R_{\mathrm{C}}$ increases. When $R_{\mathrm{C}}$ is high, marker genotyping is costly compared with phenotypic evaluation, which enhances the interest of doing replications. Despite this difference, general tendencies can be drawn from the three graphs. Like phenotypic selection, the optimal number of replications for MAS decreases with $h_{i}^{2}$ and becomes equal to one for very high $h_{i}^{2}$. For low and medium $h_{i}^{2}$, when the investment increases, the optimal number of replications first increases too. Nevertheless, unlike phenotypic selection, above a given value of investment, the optimal number of replications decreases to become equal to one (when $R_{\mathrm{C}}=1$ or 4 ) or two (when $R_{\mathrm{C}}=8$ ). This decrease is explained by the major effect of the population sample size on QTL detection and MAS efficiency. As soon as the experimental resources are sufficient, it becomes more valuable to genotype a large population sample, even poorly evaluated agronomically, than a smaller one, better evaluated. When the experimental resources are low, the population sample size that can be genotyped is small anyway and it is more efficient to increase the heritability of the trait by doing replications.

The fact that two replications instead of one are optimal when $R_{\mathrm{C}}=8$, even for high heritabilities, is due to the small impact of doing one or two replications on the population size in this case. When $I_{0}=4000$, doing one replication instead of two only increases the population sample size from 400 to $444(+11 \%)$ when $R_{\mathrm{C}}=$ 8 , while it increases the population sample size from 

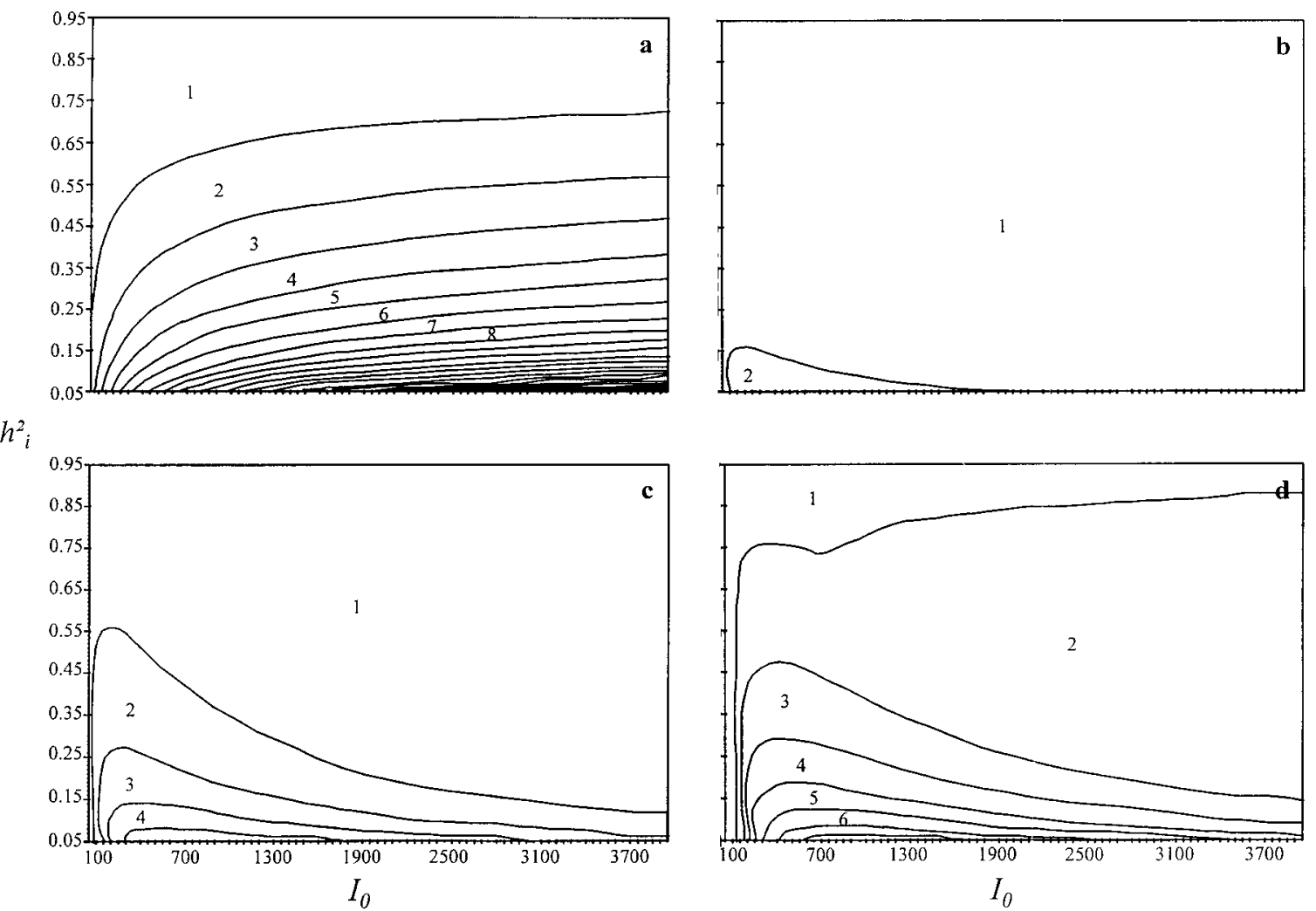

b

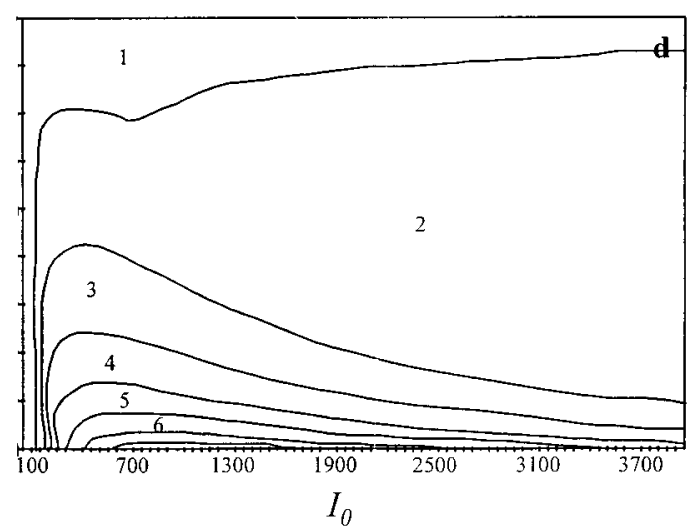

Fig. 1. Optimal number of replications for different investments, $\left(I_{0}\right.$, abscissa) and different individual heritabilities, $\left(h_{\mathrm{i}}^{2}\right.$, ordinate), for phenotypic selection (graph a) and marker-assisted selection with $R_{\mathrm{C}}$ value equal to 1 (graph b), 4 (graph c), and 8 (graph d). We assumed that 10 individuals are selected and that the determinism of the trait implies an equivalent number of 5 QTLs.

600 to $800(+20 \%)$ when $R_{\mathrm{C}}=4$, and from 1333 to 2000 $(+50 \%)$ when $R_{\mathrm{C}}=1$. Thus, for high $R_{\mathrm{C}}$ values, the gain in the selection intensity and in the power of QTL detection is small. Moreover, when $h_{i}^{2}$ is high, the phenotype is a good predictor of breeding values. The additional information that can be provided by markers is low and a moderate increase in the power of QTL detection has only a low impact on breeding value prediction and does not compensate for the broad sense heritability decrease.

The results obtained with $x_{\mathrm{e}}=10$ QTLs are not presented but shows similar trends to those observed with $x_{\mathrm{e}}=5$ QTLs, except that the optimal number of replications is slightly higher. With $x_{\mathrm{e}}=10$ QTLs, individual QTL effects are smaller. The power of QTL detection can less easily be improved by increasing the population sample size. This enhances the interest of doing replications.

For practical reasons, it is not always possible to achieve the optimal experimental design. It is thus important to evaluate the loss of efficiency associated with a non optimal number of replications. For $I_{0}=2000$, it appears that when the number of replications is close to the optimum, the loss of efficiency is generally small for both methods, especially for MAS when $R_{\mathrm{C}}>1$ and $h_{i}^{2}$ is high (Table 1$)$. When the marker genotyping is cheap $\left(R_{\mathrm{C}}=1\right)$, it is always optimal to do one replication. In this case, adding one replication reduces the population sample size (from 1000 to 667) and significantly reduces MAS efficiency. For higher $R_{\mathrm{C}}$ values, the num- ber of replications has a weaker impact on the population sample size and its effect on MAS efficiency becomes negligible near the optimum. Thus, the experimental design can be adapted to specific constraints without reducing the MAS efficiency.

Nevertheless, these results highlight that comparing the two methods of selection with a given investment leads to different designs for each one (for instance, at optimum when $h_{i}^{2}=0.15, N=222$ with 9 replications with a phenotypic selection and $N=333$ with two replications for MAS if $\left.R_{\mathrm{C}}=4\right)$. This emphasizes the importance of taking costs into account when evaluating the potential benefit of MAS.

\section{Relative Cost Efficiency of MAS}

Once the optimal number of replications for each method of selection is determined for a given global cost, the ratio of the genetic gains at optimum leads to the $R E$ of MAS. For both genetic models $\left(x_{\mathrm{e}}=5\right.$ or 10 QTLs), the general trend is towards an increase of MAS relative efficiency with $I_{0}$ and a decrease with $h_{i}^{2}$ (Fig. $2)$. When $h_{i}^{2}$ values are high, $R E$ is always below 1 . In such a case, the phenotype is a good predictor of the breeding value, the gain in accuracy provided by markers is too low to compensate for the additional experimental cost associated with the marker-genotyping. Thus, MAS is only valuable for low or medium $h_{i}^{2}$, when markers can improve the accuracy of prediction. Nevertheless, as mentioned by Moreau et al. (1998), a small 
Table 1. Genetic gains $(\Delta G)$, in genetic standard deviation unit, and population sample sizes $(N)$ corresponding to a given investment $I_{0}=2000$ but different numbers of replications (rep) with phenotypic selection and MAS, for different individual heritabilities $\left(h_{i}^{2}\right)$ and different relative costs of genotyping $\left(R_{C}\right)$. For MAS, an equivalent number of 5 QTLs is assumed.

\begin{tabular}{|c|c|c|c|c|c|c|c|c|c|c|c|c|c|c|c|c|}
\hline \multirow[b]{3}{*}{ Rep } & \multirow[b]{3}{*}{$N$} & \multicolumn{3}{|c|}{ Phenotypic selection } & \multicolumn{4}{|c|}{ MAS $\boldsymbol{R}_{\mathrm{C}}=\mathbf{1}$} & \multicolumn{4}{|c|}{ MAS $R_{C}=4$} & \multicolumn{4}{|c|}{ MAS $R_{C}=\mathbf{8}$} \\
\hline & & \multicolumn{3}{|c|}{$\Delta G$ for different $h_{i}^{2}$} & \multirow[b]{2}{*}{$N$} & \multicolumn{3}{|c|}{$\Delta G$ for different $h_{i}^{2}$} & \multirow[b]{2}{*}{$N$} & \multicolumn{3}{|c|}{$\Delta G$ for different $h_{i}^{2}$} & \multirow[b]{2}{*}{$N$} & \multicolumn{3}{|c|}{$\Delta G$ for different $h_{i}^{2}$} \\
\hline & & 0.15 & 0.30 & 0.45 & & 0.15 & 0.30 & 0.45 & & 0.15 & 0.30 & 0.45 & & 0.15 & 0.30 & 0.45 \\
\hline $\mathbf{1}$ & 2000 & 1.113 & 1.575 & 1.928 & 1000 & $2.316 \dagger$ & $2.362 \dagger$ & $2.391 \dagger$ & 400 & 1.842 & $2.026 \dagger$ & $2.070 \dagger$ & 222 & 1.364 & 1.716 & 1.822 \\
\hline 2 & 1000 & 1.352 & 1.798 & 2.085 & 667 & 2.212 & 2.259 & 2.296 & 333 & $1.914 \dagger$ & 2.002 & 2.044 & 200 & 1.587 & $1.775 \dagger$ & $1.834 \dagger$ \\
\hline 3 & 667 & 1.474 & 1.880 & $2.112 \dagger$ & 500 & 2.129 & 2.179 & 2.221 & 286 & 1.895 & 1.967 & 2.012 & 182 & 1.638 & 1.770 & 1.823 \\
\hline 4 & 500 & 1.544 & 1.908 & 2.101 & 400 & 2.061 & 2.116 & 2.160 & 250 & 1.863 & 1.932 & 1.979 & 167 & $1.644 \dagger$ & 1.753 & 1.806 \\
\hline 5 & 400 & 1.586 & $1.913 \dagger$ & 2.077 & 333 & 2.004 & 2.062 & 2.108 & 222 & 1.829 & 1.899 & 1.947 & 154 & 1.635 & 1.734 & 1.786 \\
\hline 6 & 333 & 1.611 & 1.906 & 2.048 & 286 & 1.954 & 2.015 & 2.061 & 200 & 1.797 & 1.868 & 1.916 & 143 & 1.619 & 1.713 & 1.765 \\
\hline 7 & 286 & 1.625 & 1.893 & 2.017 & 250 & 1.909 & 1.972 & 2.018 & 182 & 1.766 & 1.838 & 1.886 & 133 & 1.600 & 1.691 & 1.744 \\
\hline 8 & 250 & 1.631 & 1.876 & 1.986 & 222 & 1.869 & 1.934 & 1.980 & 167 & 1.735 & 1.809 & 1.857 & 125 & 1.580 & 1.670 & 1.722 \\
\hline 9 & 222 & $1.632 \dagger$ & 1.867 & 1.955 & 200 & 1.832 & 1.899 & 1.944 & 154 & 1.707 & 1.782 & 1.829 & 118 & 1.560 & 1.649 & 1.700 \\
\hline 10 & 200 & 1.630 & 1.837 & 1.926 & 182 & 1.797 & 1.865 & 1.910 & 143 & 1.679 & 1.755 & 1.802 & 111 & 1.540 & 1.628 & 1.678 \\
\hline
\end{tabular}

$\dagger$ Highest genetic gain obtained with the optimal number of replications. MAS = marker-assisted selection.

decrease in MAS RE can be observed for very low heritabilities because of the low power of QTL detection in this situation. MAS RE for low or medium heritabilities highly depends on the experimental resources $\left(I_{0}\right)$, especially when the relative cost of marker genotyping is high. With $x_{\mathrm{e}}=5$ QTLs and $R_{\mathrm{C}}=1$, MAS is justified in nearly all the situations (except when $h_{i}^{2}>0.75$ and $\left.I_{0}<200\right)$. When $R_{\mathrm{C}}=8$, the domain of MAS efficiency is restricted to very high $I_{0}$ values $(>1700)$ and low heritabilities. Thus, even for a low heritability, MAS could be cost inefficient if $I_{0}$ is too small. For instance, when $I_{0}=400$ and $h_{i}^{2}<0.15, R E$ is smaller than 0.6. The population sample size allowed with such an investment is too limited for the markers to improve sufficiently the accuracy of genetic value prediction. With the same investment, phenotypic selection allows breeders to do more replications, which significantly increases the broad sense heritability and thus the efficiency of the phenotypic selection.

Taking costs into account reduces the relative efficiency of MAS compared with what was found by Moreau et al. (1998). For instance, with $x_{\mathrm{e}}=5$ QTLs in
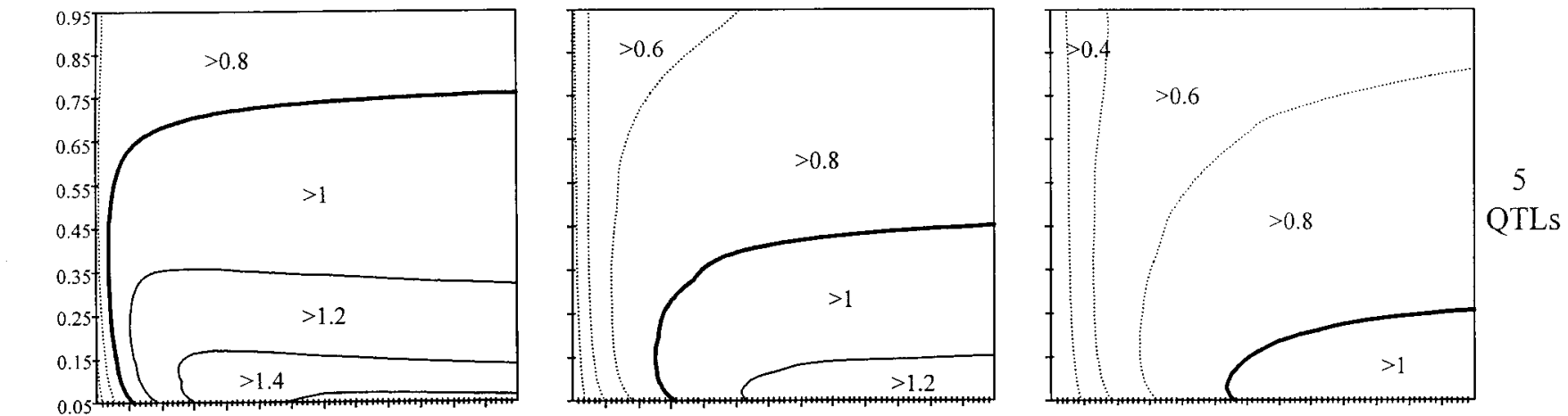

$h^{2}$
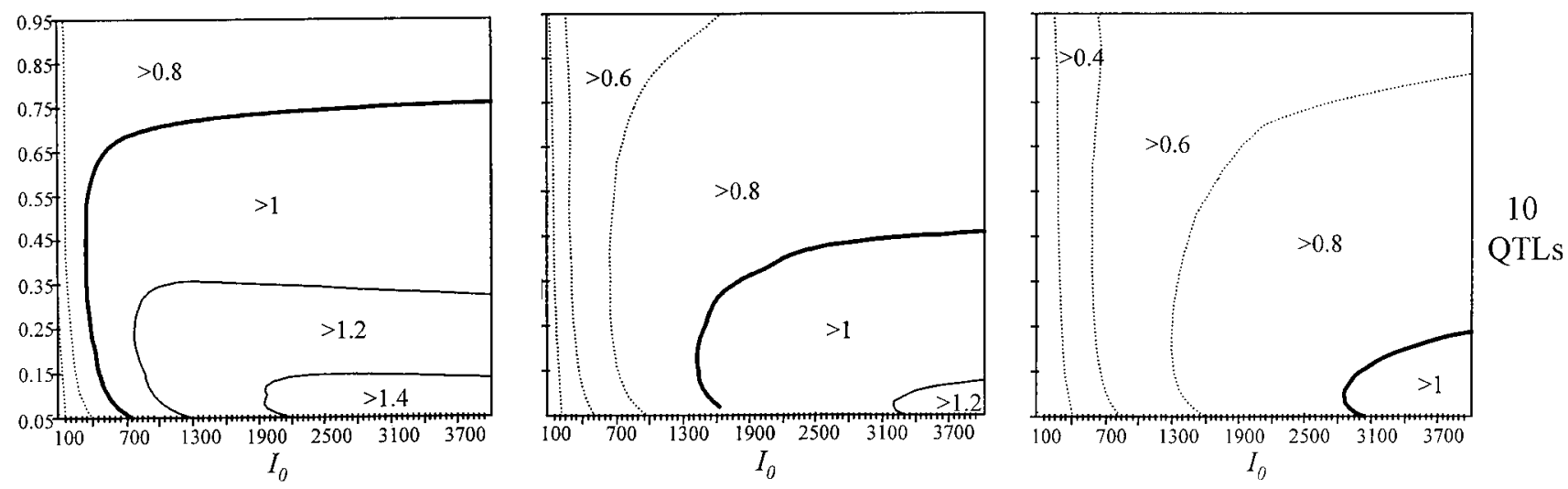

$$
R_{C}=1
$$

$R_{C}=4$

$R_{C}=8$

Fig. 2. Domains of cost efficiency of MAS for different values of the relative cost of genotyping $\left(R_{C}=1,4\right.$, or 8$)$ and two genetic models implying an effective number of 5 or 10 QTLs, as a function of the total investment $\left(I_{0}\right.$, abscissa) and the individual heritability of the trait $\left(h_{\mathrm{i}}^{2}\right.$, ordinate). 
the model, an individual heritability of 0.30 , a $R_{\mathrm{C}}$ value of 4 and an investment of 1500 , the optimal experimental design for MAS is to evaluate 300 individuals in one unreplicated trial. When phenotypic selection is assumed to be conducted with this design (i.e., without considering the problem of cost), MAS relative efficiency is expected to be 1.56 according to Moreau et al. (1998). When the phenotypic selection design is optimized for the same cost as MAS (i.e., a global investment of 1500), more individuals can be evaluated in more trials. The optimal design is to evaluate 375 individuals in four trials. With this design, the broad sense heritability becomes equal to 0.63 instead of 0.30 . The genetic gain of the phenotypic selection is increased from 1.21 (when $N=300$ and $L=1$ ) to 1.82 (when $N=375$ and $L=4$ ). As a consequence, MAS $R E$ is reduced from 1.56 to 1.04. The gap between these two results illustrates the interest of taking cost into account.

Genetic determinism affects the domains of MAS efficiency (see the upper and lower graphs in Fig. 2). MAS appears less efficient when $x_{\mathrm{e}}=10$ QTLs than when $x_{\mathrm{e}}=5$ QTLs. This result is not surprising since when more QTLs are implied in the genetic determinism, their individual effects decrease and consequently their probability of being detected. Despite the reduction in domains of MAS efficiency, the general trends observed when $x_{\mathrm{e}}=5$ QTLs are consistent with those observed when $x_{\mathrm{e}}=10$ QTLs. The differences between the two genetic situations are more striking for low $h_{i}^{2}$ values, since, in this case, the weight given to the markers in the index (Eq. [2]) is important and the power of QTL detection is a deciding parameter. When $h_{i}^{2}=0.10$, MAS requires an investment approximately twice as high when $x_{\mathrm{e}}=10$ QTLs than when $x_{\mathrm{e}}=5$ QTLs to become cost efficient.

\section{DISCUSSION}

In our study, we used simple genetic and economic models. The assumptions made for the genetic model have been discussed above and a full discussion can be found in Moreau et al. (1998). Other simplifications concern the economic part of the model. The costs of genotyping and phenotyping one individual are assumed to be independent of the population size. This is not realistic since the fixed costs linked to the necessary investment in machines, staff, etc., are the same whatever the population sample size. Thus, the cost for one data point (phenotype or marker-genotype) decreases when more individuals are analyzed simultaneously. For the agronomic evaluation, doing several trials instead of a large one is more costly, and the fixed cost associated with a trial depends on the location and the experimental conditions (for instance irrigation or not). Since all these economic aspects are difficult to quantify, they were not included in our model.

Despite the simplifications made, the results obtained with our model can be reasonably extended to real situations and highlight the major parameters that must be taken into account before undertaking a MAS experiment. When genotype $\times$ environment interactions are included in the model, it is always optimal to perform only one replication per trial. When the investment is high enough, it appears optimal to do only a small number of trials (one or two), even when genotype $\times$ environment interactions are important.

Nevertheless, such a design presents several drawbacks. Experimental problems during sowing or harvest may occur and lead to the elimination of a trial from the analysis. Traits like lodging in maize (Zea mays L.) or disease tolerances in natural conditions strongly depend on uncontrolled environmental effects and may be totally unexpressed in a given trial, in a given year. Performing only one large unreplicated trial could then be very risky. Moreover, spatial heterogeneity is more likely to occur in large field trials and without replication, it is difficult to estimate the accuracy of trials.

For these reasons, it may be less risky to increase the number of trials compared with the optimum, since it only slightly decreases the efficiency of MAS (Table 1). It may also be useful to use checks and/or to replicate a small subset of the population sample within each trial. This permits to estimate the trial accuracies and can also be used to control trial heterogeneity using spatial models, as was shown by Cullis et al. (1989) for phenotypic selection and by Moreau et al. (1999) in MAS context.

Even if a nearly optimal design may be preferred to reduce experimental risks, it still remains that the optimum for MAS is to use a reduced number of trials when compared with phenotypic selection. This strategy favors the population sample size, which is the determining parameter of MAS efficiency. This is consistent with the results found in the context of QTL detection experiments by Knapp and Bridges (1990), who showed that it is more efficient to increase the population sample size rather than the accuracy of phenotypic evaluation.

Our results show that the relative efficiency of MAS is reduced when cost is taken into account. One can further add that for low heritabilities, doing replications is not the best strategy for phenotypic selection. Breeding schemes based on performances of relatives or associated traits are more efficient. Such schemes were not considered in this study but we can infer that they would further reduce the interest of MAS (as shown by Lande and Thompson, 1990).

According to our study, the benefit of MAS in the first generation is rather small compared with the conclusions drawn by Xie and Xu (1998) or Knapp (1998). These authors do not consider the same criterion as ours to assess the cost efficiency of MAS. Roughly speaking, Knapp (1998) considers the ratio of the cost needed for MAS and phenotypic selection to achieve the same genetic gain, while Xie and Xu (1998) consider the genetic gain per cost unit of each method, both conducted with a same population size and experimental design. Moreover, Knapp (1998) evaluated the effect of the objective of selection in the comparison between MAS and phenotypic selection that was not taken into account in the other studies. Nevertheless, the difference between the results of these studies and ours mainly comes from the fact that these authors neglect the impact of 
Table 2. Maximal value of the relative cost of genotyping $\left(R_{\mathrm{C}}\right)$ below which MAS is cost efficient as a function of the individual heritability $\left(h_{i}^{2}\right)$, the number of equivalent QTLs $(5$ or 10$)$ involved in the genetic determinism of the trait and the total investment available $\left(I_{0}\right)$.

\begin{tabular}{|c|c|c|c|c|c|c|c|c|}
\hline \multirow[b]{3}{*}{$I_{0}$} & \multicolumn{8}{|c|}{$\boldsymbol{R}_{\mathrm{C}}$ limit } \\
\hline & \multicolumn{2}{|c|}{$h_{\mathrm{i}}^{2}=0.15$} & \multicolumn{2}{|c|}{$h_{i}^{2}=\mathbf{0 . 3 0}$} & \multicolumn{2}{|c|}{$h_{i}^{2}=0.45$} & \multicolumn{2}{|c|}{$h_{i}^{2}=0.60$} \\
\hline & 5 QTLs & 10 QTLs & 5 QTLs & 10 QTLs & 5 QTLs & 10 QTLs & 5 QTLs & 10 QTL \\
\hline 500 & 2 & 1 & 2 & 1 & 1 & 1 & 1 & 1 \\
\hline 1000 & 4.5 & 2.5 & 3.5 & 2.5 & 2.5 & 2 & 1.5 & 1.5 \\
\hline 1500 & 6.5 & 4 & 4.5 & 3.5 & 3 & 3 & 1.5 & 1.5 \\
\hline 2000 & 8 & 5.5 & 5 & 4.5 & 3 & 3 & 2 & 2 \\
\hline 2500 & 9.5 & 6.5 & 5.5 & 5 & 3.5 & 3.5 & 2 & 2 \\
\hline 3000 & 10.5 & 7.5 & 6 & 5.5 & 3.5 & 3.5 & 2 & 2 \\
\hline 3500 & 11.5 & 9 & 6.5 & 6 & 4 & 4 & 2 & 2 \\
\hline 4000 & 12 & 9.5 & 6.5 & 6.5 & 4 & 4 & 2 & 2 \\
\hline
\end{tabular}

MAS = marker-assisted selection.

the experimental design on QTL detection and the uncertainty of the estimated QTL effects. Even if new methods of detection based on interval mapping with a multilocus analysis (Jansen and Stam, 1994; Zeng, 1994) increase the resolution and the power of QTL detection, the assumption of known QTL effects is still optimistic. The true nature of the QTLs is still undetermined, even if genes of known function are often found to be located near the detected QTLs and provide good candidates. One can expect that progress in molecular biology or in QTL detection will help to determine more precisely the QTL positions and their effects but until now, QTL detection is still necessary. Because of sampling problems, the QTL effects estimated in one experiment are known to be biased and can hardly be inferred from QTL effects previously found in another one, even made in the same genetic background (Beavis, 1994). To limit the impact of sampling error when estimating MAS efficiency, Melchinger et al. (1998) advised the use of QTL parameters obtained from a validation experiment rather than from the original discovery experiment. This strategy is efficient for taking into account sampling bias but requires two population samples (one for QTL detection and one for validation). Thus, its benefit on the economic efficiency of MAS is difficult to evaluate. For these reasons, we find it to be more realistic to estimate the QTL effects for the first cycle in the population under selection. For the same reasons we did not consider in our comparison the case of a selection on markers only as was done by Zhang and Smith (1992) or Xie and Xu (1998). When the QTL positions are unknown, both phenotypic evaluation and complete marker genotyping of the population sample are required in the first generation and the more efficient strategy is to select individuals on an index combining markers and phenotype (Zhang and Smith, 1992).

The most important limit to the cost efficiency of MAS comes from the costs of genotyping. In maize selection, considering that 80 markers are necessary, one can estimate that the relative cost with RFLP markers (restricted fragment length polymorphism) is near 8 for usual traits like grain yield in maize ( $\$ 2$ for each marker data point and $\$ 20$ for individual phenotypic value). With such marker costs, an $R_{\mathrm{C}}$ value equal to 1 can only be achieved if the phenotypic evaluation is very expensive, around $2 \times 80=\$ 160$ per plot. Thus, for most traits, small values of $R_{\mathrm{C}}$ are certainly unrealistic and MAS interest in the first cycle is restricted to very high investments. New marker techniques, for instance microsatellites, can be automated (Schwengel et al., 1994; Perlin et al., 1995). One can expect that the improvement of marker techniques will decrease the genotyping costs and consequently increase the domains of MAS efficiency. To assess whether it is worth doing a MAS experiment, one can determine for each set of parameters $h_{i}^{2}$ and $I_{0}$, the highest $R_{\mathrm{C}}$ value that is acceptable for MAS to be cost efficient (Table 2). The maximum acceptable value to $R_{\mathrm{C}}$ increases when the individual heritability becomes lower, when the experimental resources become higher, and when the genetic determinism of the trait becomes simpler. Even with a $R_{\mathrm{C}}$ value of 8 and an investment equivalent to 3500 field plots, MAS is efficient for a complex trait when the individual heritability is equal to 0.15 . Such heritability may seem very small but it is not unrealistic for traits which are influenced by genotype $\times$ environment interactions (like stalk lodging or even yield in maize). With these parameters, the optimal design consists of evaluating 292 individuals in five trials, which is rather close to designs that are now used in QTL detection experiments.

The small benefit of MAS found in this study may be due to the fact that the evaluation of MAS was restricted to one cycle of selection for one trait. It would have been interesting to study with our model a multiple-trait selection process as was done by $\mathrm{Xie}$ and $\mathrm{Xu}$ (1998). But, the most interesting prospect may be to consider the benefit of MAS after several cycles of selection. As the relative efficiency of MAS combining markers and phenotype decreases in the course of generations (Gimelfarb and Lande, 1994; Hospital et al., 1997), we can infer that the relative economic efficiency of combined MAS after several cycles would be smaller than the one found for the first cycle. To reduce genotyping costs after the first generation, Gimelfarb and Lande (1994) proposed using combined MAS but to genotype only the markers selected in the first generation. This slightly reduces the cost of MAS but also its efficiency. It is not obvious how to predict the effect of this method on the economic efficiency of MAS. The most promising strategy seems to be the one described by Hospital et al. (1997) who showed by simulation that 
the principal interest of MAS comes from the possibility of performing two or three cycles of selection on markers only after the first cycle of combined selection, without reevaluation of the QTL effects. Once the markerQTL associations have been detected in the first cycle, the following cycles only require one to genotype individuals at the markers located near the detected QTLs. Since no phenotypic evaluation and no complete marker genotyping are needed, these cycles can be performed faster than cycles of phenotypic or combined selection and with a reduced cost. With this strategy, MAS was shown to be efficient even for medium or high heritabilities because of a best use of time, whereas the combined selection is only efficient for low heritabilities. It would be very interesting to extend our approach in this context to optimize and compare the cost efficiency of MAS with this strategy. But this requires further complex analytical developments and the use of a more realistic genetic model. We can nevertheless predict that this strategy should significantly extend the domains of cost efficiency of MAS.

\section{REFERENCES}

Beavis, W.D. 1994. The power and deceit of QTL experiments: Lessons from comparative QTL studies. p. 250-266. In D. Wilkinson, (ed.) Proc. Annu. Corn Sorghum Indus. Res. Conf., 49th, Chicago, IL. Dec 1994. Am. Seed Trade Assoc., Washington, DC.

Bos, I. 1983. The optimum number of replications when testing lines or families on a fixed number of plots. Euphityca 32:311-318.

Burrows, P.M. 1972. Expected selection differentials for directional selection. Biometrics 22:1091-1100.

Chevalet, C. 1994. An approximate theory of selection assuming a finite number of quantitative trait loci. Gen. Sel. Evol. 26:379-400.

Cullis, B.R., W.J. Lill, J.A. Fisher, J.B. Read, and A.C. Gleeson. 1989. A new procedure for the analysis of early generation variety trials. Appl. Statistics 38:361-375.

Darvarsi, A., and M. Soller. 1994. Optimum spacing of genetic markers for determining linkage between marker loci and quantitative trait loci. Theor. Appl. Genet. 89:351-357.

Falconer, D.S. 1981. Introduction to quantitative genetics. 2nd ed. Longman, New York.

Gauch, H.G., and R.W. Zobel. 1996. Optimal replication is selection experiments. Crop Sci. 36:838-843.

Gimelfarb, A., and R. Lande. 1994. Simulation of marker-assisted selection in hybrid populations. Genetical Res. 63:39-47.

Gimelfarb, A., and R. Lande. 1995. Marker-assisted selection and marker-QTL associations in hybrid population. Theor. Appl. Gen. 91:522-528.

Hospital, F., L. Moreau, A. Charcosset, and A. Gallais. 1997. More on the efficiency of marker assisted selection. Theor. Appl. Gen. 95:1181-1189.
Jansen, R.C., and P. Stam. 1994. High resolution of quantitative traits into multiple loci via interval mapping. Genetics 136:1447-1455.

Knapp, S.J. 1998. Marker-Assisted selection as a strategy for increasing the probability of selecting superior genotypes. Crop Sci. 38: $1164-1174$.

Knapp, S.J., and W.C. Bridges. 1990. Using molecular markers to estimate quantitative trait locus parameters: power and genetic variances for unreplicated and replicated progeny. Genetics 126: 769-777.

Lande, R. 1981. The minimum number of genes contributing to quantitative variation between and within populations. Genetics 99: $541-553$.

Lande, R., and R. Thompson. 1990. Efficiency of marker-assisted selection in the improvement of quantitative traits. Genetics 124 $743-756$.

Luo, Z.W., R. Thompson, and J.A. Woolliams. 1997. A population genetics model of marker-assisted selection. Genetics 146: $1173-1183$.

Melchinger, A.E., H.F. Utz, and C.C. Schön. 1998. Mapping using different testers and independent population samples in maize reveals low power of QTL detection and large biais in estimates of QTL effects. Genetics 149:383-403.

Moreau, L., A. Charcosset, F. Hospital, and A. Gallais. 1998. Markerassisted selection efficiency in populations of finite size. Genetics 148:1353-1365.

Moreau, L., H. Monod, A. Charcosset, and A. Gallais. 1999. Markerassisted selection with spatial analysis of unreplicated field trials Theor. Appl. Genet. 98:234-242.

Perlin, M.W., G. Lancia, and N. See-Kiong. 1995. Toward fully automated genotyping: Genotyping microsattelites markers by deconvolution. Am. J. Hum. Genet. 57:1199-1210.

SAS Institute Inc. 1988. SAS language: Reference. Version 6. First ed. SAS Institute Inc., Cary, NC.

Schwemgel, D.A., A.E. Jedlick, E.J. Nanthakumar, J.L. Weber, and R.C. Levitt. 1994. Comparison of fluorescence-based semi-automated genotyping of multiple microsattelite loci with autoradiographic techniques. Genomic 22:46-54.

Wricke, G., and W.E. Weber. 1986. Quantitative genetics and selection in plant breeding. Walter de Gruyter, Berlin.

Whittaker, J.C., R.N. Curnow, C.S. Haley, and R. Thompson. 1995. Using marker-maps in maker-assisted selection. Genetical Res. 66:255-265.

Whittaker, J.C., C.S. Haley, and R. Thompson. 1997. Optimal weighting on information in marker-assisted selection. Genetical. Res. 69:137-144.

Xie, C., and S. Xu. 1998. Efficiency of multistage marker-assisted selection in the improvement of multiple quantitative traits. Heredity 80:489-498.

Zeng, Z.B. 1994. Precision mapping of quantitative trait loci. Genetics 136:1457-1468.

Zhang, W., and C. Smith. 1992. Computer simulation of markerassisted selection utilizing linkage disequilibrium. Theor. Appl. Genet. 83:813-820.

Zhang, W., and C. Smith. 1993. Simulation of marker-assisted selection utilizing linkage disequilibrium: the effect of several additional factors. Theor. Appl. Genet. 86:492-496. 